\title{
O paradigma da relação entre oclusão, Ortodontia e disfunção têmporo-mandibular
}

\author{
José A. BÓSIO*
}

\begin{abstract}
Resumo
O relacionamento entre oclusão, Ortodontia e disfunção têmporo-mandibular tem sido objeto de discussões e controvérsias. No passado recente, a literatura científica mostrava que os problemas de disfunção têmporo-mandibular eram ocasionados pela má oclusão e, para sua correção, um tratamento ortodôntico deveria ser realizado. Estas teorias, aparentemente, tornaram-se obsoletas e, devido aos atuais artigos científicos mais consistentes, o relacionamento destas três entidades tem sido questionado. Esta revisão de literatura procura mostrar uma possivel mudança de paradigma quanto ao relacionamento destas três entidades.
\end{abstract}

Palavras-chave: Disfunção têmporo-mandibular. Oclusão. Ortodontia. Tratamento ortodôntico.

\section{INTRODUÇÃO}

O diagnóstico e tratamento de disfunção têmporo-mandibular (DTM) tem sido motivo de controvérsia desde a primeira vez que apareceu na literatura odontológica. E o relacionamento da DTM com duas outras entidades, Ortodontia e oclusão, apresenta uma controvérsia ainda maior.

As discussões do passado eram, na sua maioria, entre clínicos proeminentes e seus seguidores, cada um acreditando ter uma resposta objetiva para o diagnóstico e tratamento dos pacientes portadores de DTM. Diferentes especialidades dentro da odontologia defendiam diferentes conceitos para os efeitos causais e curativos. Quando Costen ${ }^{4}$ descreveu os primeiros sinais e sintomas de DTM, em 1934, vários autores passaram a acreditar em conceitos específicos sobre as causas das DTMs. Até o início dos anos 60, apenas a opinião de autores re- nomados, sem evidência científica, era o suficiente na defesa dos conceitos sobre DTM. Quando os primeiros estudos científicos consistentes apareceram, por volta dos anos 60 e 70, alguns conflitos inevitáveis surgiram, desafiando conceitos, crenças e procedimentos tradicionais.

Atualmente, uma mudança de comportamento parece estar havendo quando trata-se de disfunção têmporo-mandibular. Discussões acadêmicas estão sendo substituídas por discussões entre grupos de pesquisadores e clínicos divulgadores de hipóteses e teorias de opiniões contrárias. É possível que uma mudança na forma ou na maneira de ver este assunto possa estar acontecendo. Esta mudança faz parte de um processo evolutivo da ciência e pode mudar um paradigma².

Paradigmas nada mais são que um conjunto de modelos, formas ou padrões, e podem ser compostos

\footnotetext{
* Master of Sciences e Especialista em Ortodontia - Ohio State University, Columbus, OH. Dois anos residência em DTM na Eastman Dental Center, Rochester, NY. Especialista em DTM pelo CFO. Clínica Particular, Curitiba, PR e Concórdia, SC. Diplomado pelo American Board of Orthodontics e Board Brasileiro de Ortodontia.
} 
por várias teorias e hipóteses ${ }^{2}$. Paradigmas não podem ser questionados. Hipóteses e/ou teorias, sim. Quando uma teoria prevalente começa a ser questionada por uma nova hipótese, inicia-se uma revolução científica. A medida que a nova hipótese toma corpo, fundamentada em evidência científica, torna-se uma nova teoria. Esta nova teoria passa a ser mais discutida e difundida que a primeira, até tornar-se a teoria predominante. Conclui-se, então, o processo de revolução científica, criandose, assim, um novo paradigma, ou seja, uma nova forma de ver um determinado assunto ${ }^{2}$.

De acordo com a literatura atual, é muito provável que uma mudança de paradigma esteja acontecendo na área de DTM. Esta mudança pode encontrar resistência principalmente na postura anti-acadêmica de alguns profissionais que acreditam em suas opiniões clínicas, sem embasamento científico, e pensam que elas são mais importantes que os resultados encontrados pelos pesquisadores. ${ }^{6}$ Ainda existe uma forte corrente de profissionais disparando ataques às investigações realizadas por alguns pesquisadores, fomentando ainda mais as idéias daqueles leitores passivos, que acreditam mais na opinião de profissionais renomados do que em pesquisas bem elaboradas. Estes leitores sentem-se inibidos e tem dificuldade de abrir seus horizontes para novas filosofias e conhecimentos estabelecidos pelas novas pesquisas ${ }^{6}$.

Muitas destas novas pesquisas têm demonstrado que procedimentos oclusais, reposição das arcadas ou procedimentos cirúrgicos são, na sua maioria, agressivos e irreversíveis, além de serem excessivamente caros. Assim sendo, percebendo que seus procedimentos podem tornar-se obsoletos, estes profissionais que realizam tratamentos caros podem ficar amedrontados monetariamente ${ }^{6}$. Entretanto, alguns destes profissionais continuarão realizando estes procedimentos, talvez por desconhecer que tratamentos conservadores e/ou placebos também apresentem bons resultados. Conti ${ }^{3}$ e Greene ${ }^{6}$ mostraram que o efeito placebo, a remissão espontânea e a flutuação cíclica devem sempre ser levados em consideração quando pensamos em tratamento para disfunção têmporo-mandibular. Isberg e colaboradores $^{7}$ demonstraram que a maior parte dos pacientes com sinais e sintomas de DTM apresenta remissão espontânea após um período de cinco anos sem tratamento. Isto então significaria dizer que não devemos tratar nenhum dos nossos pacientes, pois eles vão melhorar não importando o que seja feito? Não. Alguns pacientes tem dor e não estão dispostos a esperar muito tempo para ter uma vida normal. Em alguns casos o tratamento para DTM é indicado.

Clínicos e pesquisadores deveriam estar preparados para mudar suas teorias favoritas ao longo de sua vida profissional. Seria inadmissivel aceitar e praticar apenas o que foi ensinado há trinta anos ou mais. Ao longo dos tempos, novas hipóteses e teorias aparecem e, se estiverem fundamentadas cientificamente, deveriam ser seguidas.

\section{OCLUSÃO E DTM}

Com a intenção de demonstrar que problemas intracapsulares poderiam provocar alterações oclusais, Legrell e Isberg 9 provocaram o deslocamento anterior do disco articular unilateralmente em coelhos, e mostraram que o comprimento de mandíbula era menor no lado onde houve o deslocamento de disco. Além disso mostraram também que a linha mediana estava desviada para o mesmo lado. Este resultado mostrou que a DTM intracapsular pode provocar alteração na posição da mandíbula e conseqüentemente, alterações na oclusão dentária. Com este estudo os autores determinaram um relacionamento direto de causa e efeito, onde a má oclusão não seria o fator responsável pelo problema da DTM intracapsular, mas sim o resultado do problema.

Em outro estudo, também analisando um fator oclusal específico, mordida cruzada unilateral, Santos-Pinto e colaboradores ${ }^{19}$ estudaram um grupo pequeno de pacientes ( 9 meninas e 6 meninos), e notaram que assimetrias de posição mandibular produziam assimetrias mandibulares esqueléticas, especialmente no ramo mandibular. A mandíbula estava significativamente mais longa no lado que não 
apresentava mordida cruzada. Eles sugeriram que as assimetrias esqueléticas mandibulares podem ser adaptações associadas ao ajuste postural. Este estudo, diferente do estudo de Legrell e Isberg', indica que um fator oclusal poderia ser responsável por alterações morfológicas na mandíbula, e talvez intracapsular, mesmo sem isto ter sido investigado no estudo.

Vitral e Telles ${ }^{23}$ estudaram 30 pacientes, com Classe II, subdivisão 1, de Angle, através de tomografia computadorizada e não encontraram nenhuma diferença estatisticamente significante para assimetria médio-lateral ou ântero-posterior dos processos condilares do lado com oclusão de Classe I e do lado com oclusão de Classe II. Este estudo, diferente no formato, encontrou resultados opostos aos resultados do estudo de Santos-Pinto e colaboradores ${ }^{19}$.

McNamara, Seligman e Okeson ${ }^{10}$, em uma revisão de literatura, encontraram uma baixa associação entre fatores oclusais que caracterizam DTMs. Eles mostraram que um tratamento ortodôntico realizado durante a adolescência geralmente não aumenta ou diminui a probabilidade de desenvolver DTM no futuro. E nenhum tipo de mecânica ortodôntica ou protocolo de extrações aumenta os riscos de desenvolver DTM. Eles concluíram ainda que não atingir uma oclusão gnatologicamente ideal, principal objetivo nos tratamentos ortodônticos, não significa que sinais e sintomas da DTM irão aparecer. Os autores declaram ainda que, de acordo com a literatura atual, o relacionamento de DTM com oclusão e tratamento ortodôntico é muito pequeno. Alguns fatores da oclusão, como mordida aberta anterior esquelética, overjet maior que $6-7 \mathrm{~mm}$, deslizes oclusais maiores que $4 \mathrm{~mm}$, mordida cruzada unilateral (resultados encontrados por SantosPinto e colaboradores ${ }^{19}$ ) e falta de 5 ou mais dentes posteriores podem estar associados com diagnóstico específico de DTM. Eles concluíram que os sinais e sintomas de DTM ocorrem em indivíduos com saúde normal e aumentam com a idade, principalmente durante a adolescência. Assim sendo, as disfunções têmporo-mandibulares que aparecerem durante qualquer procedimento odontológico não podem ser relacionadas ao tratamento realizado, mas sim a um fenômeno de ocorrência natural.

Os mesmos resultados foram encontrados por Pullinger e Seligman ${ }^{13}$ em um estudo recente. Eles examinaram 381 pacientes e seus respectivos modelos de gesso, tentando encontrar fatores oclusais que poderiam indicar pacientes com DTM. Eles concluíram que a oclusão dentária é responsável por apenas uma pequena parcela da amostra do estudo, não mais que $4,8 \%$ a $27,1 \%$ de probabilidade. Dos fatores oclusais relacionados, deslizes oclusais entre máxima inter-cuspidação habitual $(\mathrm{MIH})$ e posição de relação cêntrica (PRC), e overjet acentuado foram o mais alto valor predictivo para o problema de DTM intracapsular. O resultado dos últimos estudos ${ }^{10,13}$, indicam que devemos tomar muito cuidado ao dizer que problemas oclusais são a causa dos problemas de DTM. Por muitos anos esta foi a crença. Entretanto, os estudos evoluíram, e esta não é mais a teoria prevalente.

\section{ORTODONTIA E DTM}

Durante os anos 70 e 80, a crença de que a DTM era causada por má oclusão dentária era evidente. A DTM deveria então desaparecer quando eliminada a má oclusão, através de tratamento ortodôntico ou protético (mudança de esquema oclusal). No final dos anos 80 e início dos anos 90 as revisões de literatura feitas por Reynders ${ }^{15}$ e Sadowsky ${ }^{18}$ sobre o relacionamento dos problemas de articulação têmporo-mandibular e Ortodontia mostraram que nos artigos científicos consistentes, ou seja, aqueles que apresentavam grupos de estudo e de controle, não havia diferença nos sinais e sintomas de DTM entre os pacientes que eram tratados ortodonticamente e os que não eram tratados. A conclusão destes estudos foi de que: existe um relacionamento muito pequeno entre os problemas de DTM e tratamento ortodôntico. Assim sendo, a crença de que os problemas de DTM eram originados nos problemas oclusais caiu por terra. Problemas dos músculos da face e problemas intracapsulares da articulação têmporo-mandibular passaram a ser o foco prin- 
cipal das discussões. Da mesma forma, Tallents e colaboradores $^{21}$ determinaram que pode não haver uma associação forte entre relacionamento incisal, posição condilar e disfunções têmporo-mandibulares. Greene e vários outros autores ${ }^{1,6,8,10,20}$ declararam que um ortodontista prudente, antes de iniciar um tratamento ortodôntico, deveria identificar e documentar qualquer sinal ou sintoma de disfunção têmporo-mandibular. Se sintomas dolorosos aparecerem durante o tratamento, a terapia deveria ser modificada, interferências oclusais pesadas deveriam ser aliviadas e as forças, com intenção de distalizar dentes, eliminadas ou alteradas. Eles acreditam que pacientes com problemas de DTM devem ser tratados com habilidade e cuidados especiais.

Em estudos recentes sobre presença de deslocamento anterior de disco articular da ATM (verificados com exame de ressonância magnética) em voluntários assintomáticos, Tasaki e colaboradores ${ }^{22}$ encontraram uma freqüência de $29 \%$, enquanto que Ribeiro e colaboradores ${ }^{16}$ encontraram um freqüência de $34 \%$. Por outro lado, Paesani e colaboradores ${ }^{12}$ pesquisaram a incidência de deslocamento anterior de disco articular em pacientes sintomáticos, e encontraram que existe uma freqüência de $85 \%$ de distúrbios intracapsulares nestes pacientes. Estes resultados analisados conjuntamente podem indicar uma tendência em dizer que pacientes com sinais e sintomas de DTM apresentam uma freqüência maior de deslocamento de disco articular do que voluntários assintomáticos. Conseqüentemente, a explicação clínica para possíveis diagnósticos e formas de tratamento do problema seria mais fácil.

Nebbe e colaboradores ${ }^{11}$ estudaram um grupo de meninas submetidas à estimulação do crescimento mandibular. Eles sugeriram que o reposicionamento anterior de côndilo em articulações com deslocamento anterior de disco articular e onde o disco não retornava à posição normal durante o reposicionamento mandibular poderia ocasionar danos e inflamação nos tecidos da articulação. Este procedimento poderia interferir na capacidade adaptativa do tecido retrodiscal e resultar em remodelação condilar.
Neste mesmo estudo, eles mostraram ainda que existiam associações entre distúrbios intracapsulares e morfologia craniofacial. Mas nenhuma associação entre causa e efeito foi detectada. Eles também sugeriram que, durante o planejamento do tratamento ortodôntico, considerações especiais sejam dadas às condições da articulação têmporo-mandibular.

Tentando encontrar características esqueléticas que proporcionassem a identificação de pacientes portadores de distúrbios intracapsulares através de telerradiografias de perfil , Bósio e colaboradores ${ }^{1}$ estudaram 96 indivíduos, divididos em três grupos de 32 , sendo um grupo de controle (assintomático sem deslocamento de disco articular) e dois grupos de estudo (sintomático sem e com distúrbio intracapsular). Eles encontraram que o ângulo SNB igual ou menor que $75.61^{\circ}$, em pacientes portadores de Classe I de Angle, poderia ser indicativo de deslocamento anterior de disco articular. Entretanto, eles sugeriram que, sem comprovação científica com estudos prospectivos onde todas as variáveis sejam controladas, este dado deveria ser usado com cautela.

Rendell e colaboradores ${ }^{14}$, em um estudo de 18 meses de duração em pacientes ortodônticos, encontraram que a maior parte dos pacientes que iniciaram tratamento ortodôntico não desenvolveu sinais e sintomas de DTM; e os pacientes que já apresentavam algum sinal ou sintoma de DTM não apresentaram grandes mudanças na sintomatologia após o tratamento, não encontrando assim nenhuma diferença no relacionamento das duas entidades.

Ruf e Pancherz ${ }^{17}$ examinaram 20 pacientes que haviam sido tratados com aparelho de Herbst 4 anos antes da realização da pesquisa. Os exames realizados por eles foram: questionário anamnéstico, exame clínico e exame de ressonância magnética da articulação têmporo-mandibular. Analisando os resultados, eles encontraram que $25 \%$ dos pacientes examinados apresentavam sinais de disfunção têmporo-mandibular moderados ou severos e 15\% apresentavam sinais leves. Eles concluíram que o tratamento com aparelho de Herbst não parece ter resultados adversos na ATM a longo prazo. Talvez o problema na ATM já estivesse 
presente antes de ser iniciado o tratamento, pois eles não sabiam da condição intracapsular da ATM prétratamento com aparelho de Herbst.

Por outro lado, em um artigo de opinião pessoal, Sondhi ${ }^{20}$ sugeriu que, mesmo sem haver nenhuma correlação estatística entre disfunção oclusal e DTMs, em determinados pacientes, uma mudança no esquema oclusal, através de tratamento ortodôntico, poderia modificar uma disfunção intracapsular. Esta mudança no esquema oclusal deveria ocorrer sempre após a utilização de placas oclusais e após haver estabilização e redução dos sintomas de dor e estalido. Sondhi sugeriu também que seja informado ao paciente, através de formulário de consentimento informado, a possibilidade dos sintomas retornarem após a estabilização da mordida com o tratamento ortodôntico. Entretanto, Sondhi não sugere como alternativa de tratamento, após o uso de placa oclusal, a possibilidade de um ajuste oclusal para equilibrar a mordida. Talvez isto ocorra por causa de sua formação ortodôntica.

Franco e colaboradores ${ }^{5}$ estudaram 112 articulações com exame de ressonância magnética tomados antes (T1) e 18 meses após (T2) usar aparelho de Fränkel. As 56 crianças foram aleatoriamente divididas em 2 grupos: um que usou e outro que não usou o aparelho. O grupo tratado teve melhora no posicionamento do disco em todas as articulações que apresentavam deslocamento entre $\mathrm{T} 1$ e $\mathrm{T} 2$. Eles sugeriram que o tratamento com aparelho de Fränkel pode ajudar algumas crianças com disfunção têmporo-mandibular incipiente.

Kim e Graber ${ }^{8}$, em um estudo recente, objetivando avaliar o relacionamento entre disfunção têmporo-mandibular e o tratamento ortodôntico através de uma meta-análise, concluíram que devido à variedade de métodos usados nos inúmeros artigos, não foi possível realizar uma análise verdadeira devido à falta de um esquema de classificação amplamente aceito na literatura. Entretanto, eles encontraram que o tratamento ortodôntico tradicional não aumentou a prevalência de disfunção têmporo-mandibular.

\section{CONCLUSÃO}

Devido à quantidade de sinais e sintomas de disfunção têmporo-mandibular (DTM) e à falta de definição sobre o que é DTM, torna-se difícil fazer um diagnóstico específico sobre o problema. Disfunção têmporo-mandibular, uma doença multifatorial, é na realidade uma soma de termos usados para descrever sintomas de diversas causas biológicas. Somente através de descrições definitivas baseadas em evidências de fatores etiológicos individuais é que a DTM será mais efetivamente caracterizada, diagnosticada e tratada. Assim, por falta de padronização no sistema de classificação de diagnóstico e pela quantidade de artigos publicados em relação à disfunção têmporo-mandibular, sempre será possível encontrar algum artigo científico para provar um ponto de vista. Mas os materiais e métodos destes estudos devem ser considerados cuidadosamente para determinar se realmente são confiáveis. Evidências científicas significantes apontam para uma tendência de não associação do relacionamento entre tratamento ortodôntico, oclusão e disfunção têmporo-mandibular.

Aparentemente, a maior parte dos cursos de pós-graduação em Ortodontia já estão ensinando esta filosofia aos seus alunos. Mas o mesmo parece não acontecer em muitos cursos de graduação em Odontologia, que ainda insistem na tese de que o problema de disfunção têmporo-mandibular é um problema de oclusão. Oclusão, por si só, é um termo bastante abrangente e pode ser dividido em muitas variáveis. Talvez seja esta razão de encontrarmos cada vez menos artigos científicos discutindo o relacionamento entre oclusão e disfunção têmporo-mandibular.

O paradigma parece realmente estar mudando. O foco principal dos problemas de DTM está voltando-se mais para a própria articulação têmporo-mandibular. Entretanto, não devemos desprezar outros fatores associados aos problemas de DTM. 


\title{
The paradigm of the relationship among occlusion, Orthodontics and temporomandibular disorders
}

\author{
Abstract \\ The relationship between occlusion, Orthodontics and temporomandibular disorders have been subject of \\ discussions and controversies. In the past, the scientific literature stated that the temporomandibular disorders \\ occurred due to the malocclusion, and, for its correction, an orthodontic treatment should be performed. \\ Apparently, those theories have become obsoletes, and according to more consistent scientific articles, the \\ relationship among these entities have been questioned. This literature review shows a possible change in the \\ paradigm of the relationship among the three entities.
}

Key words: Occlusion. Orthodontics. Orthodontic treatment. Temporomandibular disorders.

\section{REFERÊNCIAS}

1. BOSIO, J. A.; BURCH, J. G.; TALLENTS, R. H.; WADE, D. B.; BECK, F. M. Lateral cephalometric analysis of asymptomatic volunteers and symptomatic patients with and without bilateral temporomandibular joint disk displacement. Am J Orthod Dentofacial Orthop, v. 118, p. 248-255, 1998

2. CARLSON, D. S. Craniofacial biology as Anormal science. In:_. A new vistas in orthodontics. Philadelphia: Lea \&Febiger, 1985. p. 12-37.

3. CONTI, P. C. Low level laser therapy in the treatment of TMD: a double blind pilot study. Cranio, Baltimore, v. 15, p. 144-149, 1997.

4. COSTEN, J. B. A syndrome of ear and sinus symptoms dependent upon disturbed function of the temporomandibular joint. Annals Otol Rhinol Laryngol, St. Louis, v. 43, p. 1-15, 1934

5. FRANCO, A. A. et al. Fränkel appliance theraphy and the temporomandibular disc: a prospective magnetic resonance imaging study. Am J Orthod Dentofacial Orthop, St. Louis, v. 121, p. 447-457, 2002.

6. GREENE, C. S.; MOHL, N. D.; McNEILL, C.; CLARK, G. T.; TRUELOVE, E $\mathrm{L}$. Temporomandibular disorders and sciences: a response to the critics. Am J Orthod Dentofacial Orthop, St. Louis, v. 116, p. 430-431, 1999.

7. ISBERG, A. M.; STENSTRÖEN, B. ISACSSON, G. Frequency of bilateral temporomandibular joint disc displacement in patients with unilateral symptoms: a 5-year follow-up of the asymptomatic joint. A clinical and arthrotomographic study. Dentomaxillofac Radiol, Tokyo, v. 20, p. 73-76, 1991

8. KIM, M R. GRABER, T.M Orthodontics and temporomandibular disorders: a meta-analysis. Am J Orthod Dentofacial Orthop, St. Louis, v.121, p. 438-445, 2002.

9. LEGRELL, P. E.; ISBERG, A. Mandibular length and midline asymmetry after experimentally induced temporomandibular joint disk displacement in rabbits. Am J Orthod Dentofacial Orthop, St. Louis, v. 115, p. 247-253, 1999

10. MCNAMARA, J. A.; SELIGMAN, D.; OKESON, J. Occlusion, orthodontic treatment, and temporomandibular disorders: a review. J Orofacial Pain, Carol Stream, v. 9, p. 73-90, 1995.

11. NEBBE, B.; MAJOR, P. W.; PRASAD, N. G. N. Female adolescent facial pattern associated with TMJ disk displacement and reduction in disk lenght: Part I. Am J Orthod Dentofacial Orthop St. Louis, v. 116 , p. $168-176,1999$

12. PAESANI D, WESTESSON PL, HATALA MP, TALLENTS RH AND KURITA K: Prevalence of temporomandibular joint internal derangement in patients with craniomandibular disorders. Am J Orthod Dentofacial Orthop, St. Louis, v. 101, p. 41-47, 1992.

13. PULLINGER, A. G.; SELIGMAN, D. A. Quantification and validation of predictive values of occlusal variables in temporomandibular disorders using a multifactorial analysis. J Phrostet Dent St. Louis, v. 83, p. 66-75, 2000.
14. RENDELL, J. K.; NORTON, L. A.; GAY, T. Orthodontic treatment and temporomandibular disorders. Am J Orthod Dentofacial Orthop, St. Louis, v. 101, p. 84-87, 1992.

15. REYNDERS, R. M. Orthodontics and temporomandibular disorders: a review of the literature (1966-1988). Am J Orthod Dentofacial Orthop, St. Louis, v. 97, p. 463-471, 1990.

16. RIBEIRO, R. F.: TALLENTS, R. H.; KATZBERG, R. W.; MURPHY, W. C.; MOSS, R. H.; MAGALHAES, A. C. The prevalence of disc displacement in symptomatic and asymptomatic volunteers aged 6 to 25 years. J Orofacial Pain, v. 11, p. 37-47, 1997.

17. RUF, S.; PANCHERZ, H. Long-term TMJ effects of Herbst treatment: a clinical and MRI study. Am J Orthod Dentofacial Orthop, St. Louis, v. 114, p. 475-483, 1998.

18. SADOWSKY, C. The risk of orthodontic treatment for producing temporomandibular disorders: A literature Overview. Am J Orthod Dentofacial Orthop, St. Louis, v. 101, p. 79-83, 1992.

19. SANTOS-PINTO, A.; BUSCHANG, P. H.; THROCKMORTON, G. S.; CHEN, P. Morphological and positional asymmetries of young children with functional unilateral posterior crossbite. Am J Orthod Dentofacial Orthop, St. Louis, v. 120, p. 513-520, 2001

20. SONDHI, A. Orthodontics and patients with temporomandibular disorders: Inform before you perform. Am J Orthod Dentofacial Orthop, St. Louis, v. 115, p. 551-552, 1999.

21. TALLENTS, R. H.; CATANIA, J.; SOMMERS, E. Temporomandibular joint findings in pediatric population and young adults: a critical review. Angle Orthod, Appleton, v. 61, p. 7-16, 1991.

22. TASAKI, M. M.; WESTESSON, P. L.; ISBERG, A. M.; REN, Y. F.; TALLENTS, R. H. Classification and prevalence of temporomandibular joint disk displacement in patients and symptom-free volunteers. Am J Orthod Dentofacial Orthop, St. Louis, v. 109, p. 249-262, 1996.

23. VITRAL, R. W. F.; TELLES, C. S. Computed tomography evaluation of temporomandibular joint alterations in Class II Division 1 subdivision patients: condylar symmetry. Am J Orthod Dentofacial Orthop, St. Louis, v. 121, p. 369-375, 2002.
Endereço para correspondência

José A. Bósio

Rua Marechal Deodoro, 630 Sala 1702

CEP: 80010-912 - Curitiba - PR

jbosio@bosio.odo.br / www.bosio.odo.br 